\title{
Controle automático de registros de descarga com acionamento pneumático em unidades de armazenagem agrícola
}

\author{
Automatic control of slide gate valves with pneumatic drive in agricultural storage facilities
}

Pedro Luís Andrighetto ${ }^{{ }^{*}}$ Antonio Carlos Valdiero ${ }^{\mathrm{I}}$ Maurício de Souza ${ }^{\mathrm{I}}$

\begin{abstract}
Este trabalho apresenta os resultados dos testes de um sistema de posicionamento pneumático usado no controle automático da abertura da gaveta dos registros de descarga. Esses registros são empregados para controlar o carregamento dos grãos por gravidade nos equipamentos de transporte nas unidades de beneficiamento e armazenagem de grãos, tais como os elevadores de caçamba e as correias transportadoras. Os experimentos permitiram avaliar o desempenho do posicionamento da gaveta de um protótipo de registro de descarga, acionada por um cilindro pneumático cuja posição é controlada por um controlador eletrônico do tipo ON-OFF. Nos testes mediu-se a resposta transitória da posição da gaveta a uma mudança em degrau no sinal de referência e foram avaliados o erro de posição, o sobrepassado e a velocidade. Os resultados mostraram a viabilidade do sistema no desempenho do controle da posição, na simplicidade do acionamento e no baixo custo, sendo uma alternativa para automação de instalações agroindustriais.
\end{abstract}

Palavras-chave: engenharia agrícola, máquinas agrícolas, pós-colheita.

\section{ABSTRACT}

This paper shows the results obtained from tests of a pneumatic positioning system used in the automatic control of the drawer opening in slide gate valves. They are used to control the grain loading by gravity in transport equipments as bucket elevators and belt conveyors in agricultural storage facilities. Experimental tests were carried out to evaluate the performance of drawer positioning in a prototype of slide gate valve that is driven by a pneumatic cylinder with position control carried out by an ON-OFF electronic controller. In the experiments was measured the step response of drawer position,

\begin{abstract}
allowing the evaluation of the position error, the overshoot and the velocity. The results show the viability of this solution considering its performance in position control, drive simplicity and low cost. It represents an alternative to automation of agricultural storage facilities.
\end{abstract}

Key words: agricultural engineering, agricultural machines, postharvest.

Os registros de descarga (Figura 1A) são equipamentos usados nas unidades de beneficiamento e armazenagem para o controle do carregamento dos grãos por gravidade nos equipamentos de transporte, como, por exemplo, elevadores de caçamba e correias transportadoras, por meio da abertura/do fechamento de uma gaveta. A figura 1B mostra um exemplo de aplicação em que um registro de descarga é instalado na parte inferior de um silo pulmão para controlar o fluxo de grãos descarregados em uma correia transportadora, cuja vazão mássica é controlada pela área de abertura da gaveta (Figura 1A).

A importância do controle automático da posição de abertura da gaveta do registro de descarga é evidenciada na figura $1 \mathrm{~B}$, pois uma abertura exagerada pode causar um fluxo excessivo de grãos, sobrecarregando a correia transportadora. Conforme FEHLBERG (2005), isso pode ocasionar queima do motor elétrico de acionamento, redução da vida útil do motor devido ao superaquecimento por excesso de

IDepartamento de Tecnologia, Campus Panambi, Universidade Regional do Noroeste do Estado do Rio Grande do Sul (UNIJUí). Av. Rudi Franke, 540, 98280-000, Panambi, RS, Brasil. E-mail: pedro@unijui.edu.br.*Autor para correspondência. 

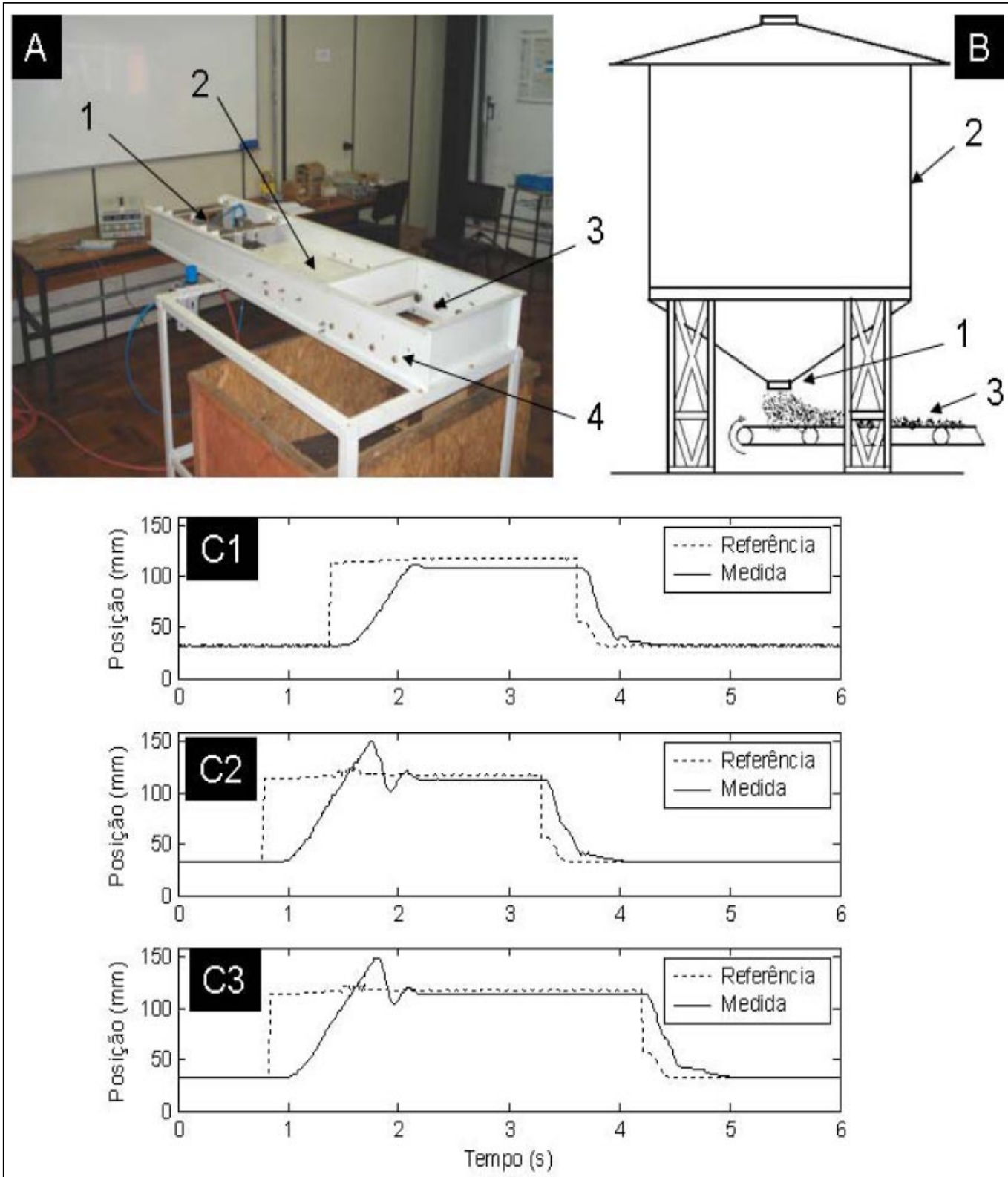

Figura 1 - Registro de descarga com acionamento pneumático: protótipo testado (A), destacando-se o sistema pneumático (A1), a gaveta totalmente aberta (A2), a área de passagem de grãos máxima (A3) e a estrutura suporte (A4); aplicação do registro de descarga (B1) em um silo pulmão (B2) para o carregamento de uma correia transportadora (B3) e os resultados dos testes para a posição da gaveta do registro de descarga em função do tempo: resultado para pressão de suprimento 4,0bar (C1), resultado para pressão de suprimento 5,0bar (C2) e resultado para pressão de suprimento 6,0bar (C3).

corrente ou ainda problemas de funcionamento na instalação agroindustrial, como o embuchamento de equipamentos após o registro e o desalinhamento de correias transportadoras. Estas falhas podem interromper o processo produtivo e o recebimento de cereais em plena safra, trazendo prejuízos aos fabricantes dos equipamentos, às empresas armazenadoras e aos produtores agrícolas. O problema da sobrecarga pode ser resolvido com o controle automático da abertura do registro em função da corrente exigida pelo motor elétrico da correia transportadora medida por meio de sensores como os transformadores de corrente (TC). Havendo fluxo excessivo, a corrente no motor é maior que a nominal e o registro de descarga tem sua abertura reduzida, diminuindo o fluxo de grãos e eliminando a sobrecarga. 
Um fator relevante para a competitividade no setor de equipamentos para armazenagem agrícola é a constante evolução tecnológica, principalmente envolvendo a automação de equipamentos. Conforme MARTINELLI \& SOUZA (2003), em países desenvolvidos, constata-se uma maior automação dos silos e dos demais equipamentos utilizados na armazenagem de grãos. No Brasil, há poucos trabalhos publicados sobre automação no setor de armazenagem agrícola, sendo que os trabalhos são concentrados basicamente em aeração de grãos (SILVA, 2004). Outros autores destacam os esforços tecnológicos para automação da secagem de grãos (BROD \& DEL FABBRO, 2005). Para a automação do carregamento dos equipamentos de transporte, FEHLBERG (2005) desenvolveu o projeto de um registro de descarga com acionamento por motor elétrico e redutor. Esse registro necessita de um mecanismo do tipo pinhão/cremalheira para transformar o movimento de rotação do motor elétrico em movimento de translação da gaveta, sensores indutivos de fim de curso e quadro com disjuntores e relés de acionamento, tornando-se uma solução cara em componentes e custo de montagem. Neste trabalho foi estudada uma solução mais simples e barata: a aplicação de um cilindro pneumático no controle da posição da gaveta do registro, mas que apresenta vários problemas de controle causados pelo forte comportamento não-linear oriundo da compressibilidade do ar, da força de atrito nos cilindros e da vazão nas válvulas de controle (ANDRIGHETTO et al., 2003).

A importância da realização, da discussão e do relato de testes para o desenvolvimento de equipamentos agrícolas foi evidenciada por diversos autores (ALONÇO et al., 2006; BORTOLAIA et al., 2008). Assim sendo, o objetivo deste trabalho foi testar e avaliar o desempenho de um sistema de posicionamento pneumático no controle automático da abertura da gaveta de registros de descarga, contribuindo com o desenvolvimento de uma solução inovadora para a automação de instalações armazenadoras agrícolas.

O protótipo usado nos testes (Figura 1A) é composto por uma estrutura suporte, uma gaveta, um sistema pneumático, um sensor de posição da gaveta e um controlador eletrônico. A gaveta tem largura e curso iguais a $400 \mathrm{~mm}$ e pode assumir qualquer posição de abertura entre 0 e 400mm. O acionamento pneumático é formado por um cilindro de dupla ação, com diâmetro de $40 \mathrm{~mm}$ e curso de $400 \mathrm{~mm}$, uma válvula direcional com cinco vias, três posições e posição central fechada, acionada por duas bobinas 24V CC, um conjunto filtro-regulador de pressão e duas válvulas reguladoras de vazão. Um potenciômetro linear é usado para medir a posição da gaveta, fornecendo um sinal de medição $V_{p}$ de 0 a 9,0V. O controlador eletrônico é do tipo ON-OFF e foi implementado com amplificadores operacionais. O erro de posição é definido como a diferença entre o sinal de referência $V_{R}$ e $V_{P}$, sendo o seu valor máximo definido nesta aplicação como $22,5 \mathrm{~mm}$, verificado por meio de resistores no controlador.

Nos testes foi obtida a resposta transitória da posição da gaveta a uma mudança em degrau no sinal de referência $V_{R}$. Este tipo de teste é muito usado na avaliação de sistemas de controle porque simula satisfatoriamente uma das situações mais comuns de funcionamento, que é uma mudança brusca no sinal de referência (CARVALHO, 2000). Os testes C1, C2 e C3 (Figura 1C) foram feitos sem grãos nas pressões de suprimento do ar comprimido $\left(\mathrm{P}_{\mathrm{s}}\right)$ de 4,0bar (Teste C1), 5,0bar (Teste C2) e 6,0bar (Teste C3), buscando avaliar o desempenho do acionamento em pressões diferentes, situação que pode ocorrer na prática. Os testes iniciam com a gaveta totalmente fechada $\left(V_{R}=0 \mathrm{~V}\right)$, então $V_{R}$ é alterada em degrau para $2,5 \mathrm{~V}$, que corresponde a uma abertura de cerca de $90 \mathrm{~mm}$ na posição da gaveta e a uma área de passagem de grãos de $0,036 \mathrm{~m}^{2}$. Após manter a gaveta posicionada por cerca de $2 \mathrm{~s}, \mathrm{~V}_{\mathrm{R}}$ é novamente conduzida em degrau a $0 \mathrm{~V}$, fechando a gaveta.

A posição da gaveta foi registrada por um sistema de aquisição de sinais por computador, à taxa de amostragem de 10ms e tempo total de aquisição 6 s. A velocidade de abertura da gaveta $\left(\mathrm{V}_{\mathrm{a}}\right)$ foi calculada a partir dos dados retirados de um trecho do gráfico da posição em função do tempo (Figura 1C), em que a variação da posição se aproxima de uma linha reta. Essa variação (em mm) é dividida pelo intervalo de tempo (em s) correspondente, obtendo-se a velocidade. O erro de posição $\left(E_{p}\right)$ foi calculado com a gaveta aberta, como a diferença entre a posição de referência e a posição da gaveta após haver o posicionamento. O sobrepassado $\mathrm{S}_{\mathrm{p}}$ (overshoot) foi calculado como a diferença entre a abertura máxima e a posição final da gaveta.

A figura 1C mostra os resultados para a posição da gaveta em função do tempo do experimento. No teste $C 1$, obteve-se $S_{p}=3,3 \mathrm{~mm}$. A velocidade $V_{a}$ é igual a 166,1 $\mathrm{mm} \mathrm{s}^{-1}$, calculada entre 1,9 e 2,0s e $\mathrm{E}_{\mathrm{p}}^{\mathrm{a}}$ é igual a $9,2 \mathrm{~mm}$. No teste C2, obteve-se $\mathrm{S}_{\mathrm{p}}=36,2 \mathrm{~mm}$ em t $=1,75 \mathrm{~s}$. A velocidade $\mathrm{V}_{\mathrm{a}}$ é igual a $170,9 \mathrm{~mm} \mathrm{~s}^{-1}$, calculada entre 1,2 e 1,5s e $E_{p}$ é igual a 5,5mm. No teste C3, obtevese $S_{p}=34,4 \mathrm{~mm}$ em $\mathrm{t}=1,80 \mathrm{~s}$. A velocidade $\mathrm{V}_{\mathrm{a}}$ é igual a $178,9 \mathrm{~mm} \mathrm{~s}^{-1}$, calculada entre 1,3 e $1,5 \mathrm{~s}$ e $\mathrm{E}_{\mathrm{p}}$ é igual a 4,2mm. 
A análise dos testes mostrados na figura $1 \mathrm{C}$ permite verificar que a velocidade $\mathrm{V}$ tem um pequeno acréscimo com o aumento da pressão de suprimento de ar comprimido devido ao aumento da vazão de ar em função do aumento da pressão. Constatou-se um pequeno atraso de cerca de 0,2 s para o início do movimento da gaveta após o degrau no sinal de referência $\mathrm{V}_{\mathrm{R}}$ ocasionado pelo tempo necessário para o enchimento dos tubos de ar comprimido após a abertura da válvula e o estabelecimento da pressão necessária para mover o cilindro pneumático. O tempo total de posicionamento obtido nos testes foi inferior a $1,5 \mathrm{~s}$, sendo considerado satisfatório para esta aplicação.

As oscilações e o sobrepassado $\left(\mathrm{S}_{\mathrm{p}}\right)$ verificados na abertura da gaveta resultam do baixo amortecimento dos atuadores pneumáticos (ANDRIGHETTO et al., 2003). O valor de $S_{p}$ é baixo para o teste C1, enquanto que, para os testes C2 e C3, o valor encontrado é cerca de dez vezes maior, o que é causado pela força pneumática maior no cilindro quando a pressão de suprimento é maior. No fechamento da gaveta, não foi verificado sobrepassado porque o cilindro pneumático avança totalmente e entra na região de ação do seu sistema de amortecimento de final de curso. A presença do sobrepassado não é considerada crítica nesta aplicação, pois a gaveta terá uma abertura maior do que a desejada por um curto intervalo de tempo, sendo rapidamente conduzida à posição desejada com erro reduzido. Foi verificada a importância de especificar o cilindro pneumático com sistema de amortecimento nas duas posições de fim de curso, para diminuir os efeitos de choques da gaveta com o corpo do registro de descarga. Os melhores resultados de posicionamento foram obtidos com a pressão de suprimento de 6bar (Teste C3), sendo a recomendada para esta aplicação, além de proporcionar uma maior força pneumática para o acionamento da gaveta.

Os valores de $\mathrm{E}_{\mathrm{p}}$ obtidos nos testes foram inferiores ao valor máximo definido no controlador ONOFF, sendo que o aumento da pressão ocasionou uma redução nos valores de $\mathrm{E}_{\mathrm{p}}$. Verificou-se que os valores obtidos para $\mathrm{E}_{\mathrm{p}}$ não são críticos para o funcionamento do registro de descarga, tornando viável a aplicação do controlador estudado. Além disso, os valores são da mesma ordem de grandeza dos obtidos por ANDRIGHETTO et al. (2003) no teste de um controlador proporcional $(\mathrm{P})$ em um sistema de posicionamento pneumático com válvula proporcional direcional pneumática.

Os resultados experimentais permitem concluir que o uso de acionamentos pneumáticos com controladores ON-OFF é uma solução viável e de baixo custo para a automação dos registros de descarga nas instalações agroindustriais. Os valores experimentais para o erro de posição, o sobrepassado e as velocidades da gaveta atendem os requisitos necessários para uma aplicação em uma instalação agrícola.

\section{AGRADECIMENTOS}

Os autores agradecem à Fundação de Amparo à Pesquisa do Rio Grande do Sul (FAPERGS), pela concessão dos recursos financeiros necessários à execução deste Projeto de Pesquisa por meio do Edital 001/2005.

\section{REFERÊNCIAS}

ALONÇO, A.S. et al. Avaliação técnica de uma máquina para a correção de microrrelevo do solo de áreas destinadas ao cultivo de arroz irrigado. Ciência Rural, v.36, n.5, p.1643-1646, 2006.

ANDRIGHETTO, P.L. et al. Experimental comparisons of the control solutions for pneumatic servo actuators. In: COBEM 2003 - Brazilian Congress of Mechanical Engineering, 17., 2003, São Paulo, SP. Proceedings...Rio de Janeiro: Associação Brasileira de Engenharia e Ciências Mecânicas, 2003. 1 CD.

BORTOLAIA, L.A. et al. Avaliação técnica de um transportador pneumático de grãos por aspiração. Ciência Rural, v.38, n.2, p.526-529, 2008.

BROD, F.P.R; DEL FABBRO, E.L. Secos e bem armazenados. Pelotas: Grupo Cultivar, 2005. 10p. (Caderno Técnico Máquinas e Mecanização, 45).

CARVALHO, J.L.M. Sistemas de controle automático. Rio de Janeiro: LTC, 2000. 391p.

FEHLBERG, A. Controle de fluxo de carregamento de equipamentos agrícolas utilizando controladores $\mathrm{ON}$ / OFF. 2005. 61f. Trabalho de Conclusão de Curso (Graduação em Engenharia Elétrica) - Universidade Regional do Noroeste do Estado do Rio Grande do Sul.

MARTINELLI, O.; SOUZA, J.M. Relatório setorial preliminar - Setor armazenagem agrícola. Rio de Janeiro: FINEP, 2003. Capturado em 30 de jul. de 2007. Online. Disponível na Internet em: http://www.finep.gov.br/PortalDPP/ relatorio_setorial/impressao_relatorio.asp?lst_setor=18.

SILVA Jr., A.N. Eficiência energética em sistemas de aeração de silos de armazenagem de grãos, utilizando inversores de freqüência. 2004. 132f. Tese (Doutorado em Agronomia) - Faculdade de Ciências Agronômicas da UNESP, Universidade Estadual Paulista "Júlio de Mesquita Filho". 